\title{
Skin hydration of the heel with fissure in patients with diabetes: a cross-sectional observational study
}

This article was published in the following Dove Press journal: Chronic Wound Care Management and Research

\author{
Makoto Oe' \\ Kimie Takehara ${ }^{2}$ \\ Hiroshi Noguchi ${ }^{3}$ \\ Yumiko Ohashi ${ }^{4}$ \\ Mayu Fukuda' \\ Takashi Kadowaki ${ }^{5}$ \\ Hiromi Sanada ${ }^{1,6}$ \\ 'Global Nursing Research Center, \\ 2Department of Advanced Nursing \\ Technology, ${ }^{3}$ Department of Life \\ Support Technology (Molten), \\ Graduate School of Medicine, The \\ University of Tokyo, ${ }^{4}$ Department \\ of Nursing, The University of Tokyo \\ Hospital, ${ }^{5}$ Department of Diabetes \\ and Metabolic Diseases, ${ }^{6}$ Department \\ of Gerontological Nursing/Wound \\ Care Management, Graduate School \\ of Medicine, The University of Tokyo, \\ Tokyo, Japan
}

Purpose: Foot fissure should be prevented in patients with diabetes due to the likelihood of subsequent diabetic ulcer. The purpose of this study was to investigate a cutoff point for skin hydration with fissure and the factors associated with low skin hydration in patients with diabetes. Subjects and methods: Subjects were patients with diabetes who visited the diabetic foot clinic and were evaluated for skin hydration on the heel between April 2008 and March 2015. Information about fissure, skin hydration, age, sex, autonomic neuropathy, angiopathy, and tinea pedis were collected from the medical charts. Skin hydration on the heel was measured using a moisture checker. Skin hydration was compared between heels with and without fissure, and a cutoff for skin hydration with fissure was determined using receiver operating characteristic analysis. Based on the determined cutoff, factors associated with lower skin hydration were analyzed using logistic regression analysis.

Results: Participants comprised 693 patients. Mean \pm SD age was $66.8 \pm 10.8$ years, and $57.0 \%$ of subjects were male. The frequency of fissures on the heels was $10.4 \%$. Area under the receiver operating characteristic curve for skin hydration in the presence of fissure was 0.717 . Twenty percent was selected as the cutoff point, offering sensitivity of 0.478 and specificity of 0.819 . Logistic regression analysis showed correlations between three factors (male sex, tinea pedis, and room temperature) and skin hydration $<20.0 \%$ (odds ratio [OR] 1.587, 95\% CI 1.157-2.178, $p=0.004$; OR $1.548,95 \%$ CI $1.122-2.135, p=0.005$; and OR $0.900,95 \%$ CI $0.823-0.0985$, $p=0.021$, respectively).

Conclusion: To prevent heel fissures, moisturizing care should aim at achieving skin hydration of $20 \%$. If skin hydration is $<20 \%$, prevention of fissures may warrant not only specialized moisturizing care but also consideration of treatment for tinea pedis.

Keywords: xerosis, diabetes mellitus, tinea pedis, moisturizing care

\section{Introduction}

Diabetic foot is one of the complications of diabetes mellitus, representing infection, ulceration, or destruction of tissues of the foot associated with neuropathy and/or peripheral artery disease in the lower extremity of people with diabetes. ${ }^{1}$ Once diabetic foot develops, physical condition, long-term prognosis, ${ }^{2,3}$ and quality of life $\mathrm{e}^{4,5}$ are severely affected. The number of patients with diabetes is estimated to increase, ${ }^{6}$ and establishment of preventive care for diabetic foot is, therefore, an urgent issue.

Prevention of foot fissure is important in patients with diabetes, as this pathology represents one of the risk factors for developing foot ulcer. ${ }^{7}$ Fissures are generally considered to develop with dry skin resulting from decreased perspiration associated with autonomic neuropathy.
Correspondence: Makoto Oe Global Nursing Research Center, Graduate School of Medicine, The University of Tokyo, 7-3-I Hongo, Bunkyo-ku, Tokyo II 3-0033, Japan Tel +8I $3584 \mid 3605$

Fax +81358413419

Email moe-tky@umin.ac.jp 
The xerosis assessment scale, ${ }^{8-11}$ overall cutaneous score, ${ }^{9,10}$ dryness area severity index score, ${ }^{12}$ transepidermal water loss, ${ }^{13}$ and skin hydration ${ }^{13}$ have been used as indicators of dry skin. Skin hydration is affected by the amount of moisture in the stratum corneum, and can be used to quantitatively assess the severity of dry skin. However, the optimal cutoff for skin hydration to avoid fissure is unknown. The indications in terms of skin hydration for the application of moisturizer to address dry skin are thus unclear.

Skin hydration is also affected by age and climate. In addition, the presence of deep fissures correlates with angiopathy, ${ }^{14}$ although the relationship between angiopathy and skin hydration is unknown. Effective care can be established if factors associated with low skin hydration in patients with diabetes can be elucidated.

The purpose of this study was to investigate the cutoff point of skin hydration with fissure and factors associated with low skin hydration in patients with diabetes. Our findings are expected to contribute to preventive care for foot fissure in patients with diabetes.

\section{Subjects and methods Subjects}

This study was designed as a cross-sectional chart review. Subjects were patients with diabetes who visited the diabetic foot clinic at a university hospital in Tokyo and evaluated skin hydration on the heel between April 2008 and March 2015. Patients with ulcers or foot defects due to amputation were excluded. When visiting the diabetic foot clinic more than twice in this period, data from the first visit were used for analysis. The diagnosis of diabetes mellitus was based on the Japan Diabetes Society criteria. ${ }^{15}$

\section{Investigation items}

Data about fissures, skin hydration on the heel, age, sex, type of diabetes, duration of diabetes, concentration of glycated hemoglobin, coefficient of variation of R-R intervals (CVRR), ankle-brachial index (ABI), toe-brachial index (TBI), monofilament test, and tinea pedis were collected from the medical charts.

Fissure was defined as any linear gap or slit in the skin surface, ${ }^{16}$ and was judged by a certificated diabetic care nurse and a nursing researcher specializing in wound care. Only the fissures on the heel were used for analysis because the relationship between presence of fissures and skin hydration on the heel was investigated.

Skin hydration on the heel was measured using a moisture checker (TSMY-707S; Scalar Corporation, Tokyo, Japan).
The TSMY-707S is a portable device for hand-held use, and can estimate the moisture content to a depth of several dozen microns based on the capacitance in the area in contact with the sensor. Range of reading and accuracy are $0 \%-99.9 \%$ and $\pm 0.2 \%$, respectively. Measurements were made three times in the center of the heel pad, and the median was calculated. Skin hydration on either the fissure side or the side with lower skin hydration between the right foot and the left foot was used for analysis. The heel was chosen as the site of measurement because this is a site at which fissures commonly develop.

Autonomic neuropathy was assessed by CVRR. To measure variations in CVRR, a device measuring blood pressure pulse wave (form PWV/ABI BP-203RPE II; Omron Colin, Tokyo, Japan) was used. The definition of abnormal CVRR was based on in-hospital criteria: age 20-29 years, $<2.46$; $30-39$ years, $<2.13 ; 40-49$ years, $<1.66 ; 50-59$ years, $<1.41$; $60-69$ years, $<1.25$; and $\geq 70$ years, $<1.14$. Angiopathy was assessed by ABI and TBI measured in a supine position after $>15$ min of rest using the same device employed for measurement of CVRR. If $A B I$ was $\leq 0.9$, or if $\mathrm{ABI}$ was $>1.4$ and $\mathrm{TBI}$ was $<0.7$, the patient was judged as showing angiopathy. ${ }^{17}$

Regarding the Semmes-Weinstein monofilament test, plantar aspects of the first toe, first metatarsal head, and fifth metatarsal head were tested on both the feet using the $10 \mathrm{~g}$ Semmes-Weinstein monofilament. The monofilament test was repeated twice at the same site, in addition to at least one "mock" monofilament application. Sensation was determined to be normal at each site if the patient correctly answered two out of three applications.

Tinea pedis was judged from the microscopic findings of scales and hyperkeratosis on the sole by a certificated diabetic care nurse and a nursing researcher specializing in wound care. Subjects receiving treatment for tinea pedis were also judged positively for the presence of tinea pedis.

\section{Statistical analysis}

Continuous data are expressed as mean \pm SD. Differences in skin hydration between heels with and without fissure were analyzed using the independent $t$-test, and cutoffs for skin hydration with fissure were investigated using receiver operating characteristic analysis. Based on the determined cutoff for skin hydration, relationships between lower skin hydration and each parameter were analyzed using the $t$-test and chi-squared test, and factors associated with lower skin hydration were analyzed using logistic regression analysis.

Statistical analysis was performed using IBM SPSS Statistics version 20 (IBM Corporation, Armonk, NY, USA). The level of statistical significance was $p=0.05$. 


\section{Ethical consideration}

This study was approved by the ethics committee at the Graduate School of Medicine and Faculty of Medicine, University of Tokyo (No. 3078-(2)). This protocol was described in the website of the University of Tokyo and displayed on posters throughout the hospital, and subjects were provided with the opportunity to opt out.

\section{Results}

\section{Subjects}

A total of 693 subjects were included in this study. Mean \pm SD age was $66.8 \pm 10.8$ years, and $57.0 \%$ were male (Table 1 ). Autonomic neuropathy was seen in 56 subjects $(8.1 \%)$, and angiopathy was evident in $46(6.7 \%)$. Fissures on the heels were observed in 72 subjects $(10.4 \%)$.

\section{Skin hydration cutoff for fissure}

The $t$-test revealed that skin hydration was significantly lower in heels with fissure than in heels without $(14.6 \% \pm 6.3 \%$ vs. $19.1 \% \pm 6.0 \%, p<0.001)$.

Results of receiver operating characteristic analysis of skin hydration according to the presence of fissure showed an area under the curve of 0.717 ( $p<0.001$; Figure 1). Twenty percent was selected as the cutoff, offering sensitivity of 0.478 and specificity of 0.819 , because higher specificity was considered preferable for fissure prevention (Table 2).

Table I Characteristics of subjects

\begin{tabular}{ll}
\hline Variables & $\mathbf{N}=693$ \\
\hline Age (years) & $66.8 \pm 10.8$ \\
Sex & $395(57.0)$ \\
$\quad$ Male & $298(43.0)$ \\
$\quad$ Female & \\
Type of diabetes & $30(4.3)$ \\
$\quad$ Type I & $635(91.8)$ \\
Type 2 & $28(3.9)$ \\
Others & $14.3 \pm 9.9$ \\
Duration of diabetes (years) ${ }^{\mathrm{a}}$ & $7.2 \pm 1.1$ \\
HbAlc (\%) & \\
Autonomic neuropathyc & $56(8.1)$ \\
$\quad$ Yes & $635(91.9)$ \\
No & \\
Angiopathy & \\
Yes & $46(6.7)$ \\
No & $640(93.3)$ \\
Monofilament test & \\
Abnormal & $147(21.2)$ \\
Normal & $545(78.8)$ \\
\hline
\end{tabular}

Notes: $n$ (\%), mean \pm SD. Some data were missing. ${ }^{a} n=689$; ${ }^{b} n=630$; ${ }^{c}$ decreased ECG $\mathrm{R}-\mathrm{R}$ interval variability; ${ }^{\mathrm{d}}$ ankle-brachial index $\leq 0.9$, or ankle-brachial index $>\mathrm{I} .4$ and toe-brachial index $<0.7{ }^{\mathrm{e}} \mathrm{n}=692$.

Abbreviations: ECG, electrocardiogram; HbAlc, glycated hemoglobin.

\section{Factors associated with skin hydration $<20.0 \%$}

Various factors between subjects with $<20.0 \%$ of skin hydration and subjects with $\geq 20.0 \%$ of skin hydration were compared. Univariate analysis revealed that male sex and tinea pedis were significantly more common among subjects with skin hydration $<20.0 \%$ (63.1\% and $67.4 \%$, respectively) than among those with skin hydration $\geq 20.0 \%(49.4 \%, p<0.001$ and $54.5 \%, p=0.001$, respectively), as shown in Table 3. In addition, room temperature when measuring skin hydration was significantly lower among subjects with skin hydration $<20.0 \%\left(24.8^{\circ} \mathrm{C} \pm 2.0^{\circ} \mathrm{C}\right)$ than among those with skin hydration $\geq 20.0 \%\left(25.2^{\circ} \mathrm{C} \pm 1.7^{\circ} \mathrm{C}, p=0.005\right)$.

Logistic regression analysis showed correlations between three factors (male sex, tinea pedis, and room temperature) and skin hydration $<20.0 \%$ (odds ratio [OR] 1.587, 95\% CI 1.157-2.178, $p=0.004$; OR 1.548, 95\% CI 1.122-2.135, $p=0.005$; and OR $0.900,95 \%$ CI $0.823-0.0985, p=0.021$, respectively), even though age was entered as an adjusting factor (Table 4).

\section{Discussion}

The present study is the first to seek to clarify a suitable cutoff for skin hydration in patients with diabetes at risk of heel fissure, revealing a value of $20 \%$. Furthermore, male sex, tinea pedis, and room temperature were, for the first time, identified as factors associated with skin hydration $<20 \%$. These findings may be important in designing preventive care for patients at risk of fissures.

\section{Factors associated with skin hydration $<20.0 \%$}

One of the factors associated with skin hydration $<20.0 \%$ was tinea pedis. To the best of our knowledge, a relationship between tinea pedis and skin hydration has not been previously described. Dry skin is a symptom of tinea pedis, and fissures can develop from dry skin. Direct microscopy was not conducted for the diagnosis of tinea pedis in this study. Further research using direct microscopy is thus needed to validate the relationship between lower skin hydration and tinea pedis.

Male sex and lower room temperature were also found as factors associated with skin hydration $<20.0 \%$. To the best of our knowledge, the relationships between male sex or room temperature and skin hydration have not yet been clarified. Confounding factors such as application of moisturizer and season might thus influence the results of studies. 


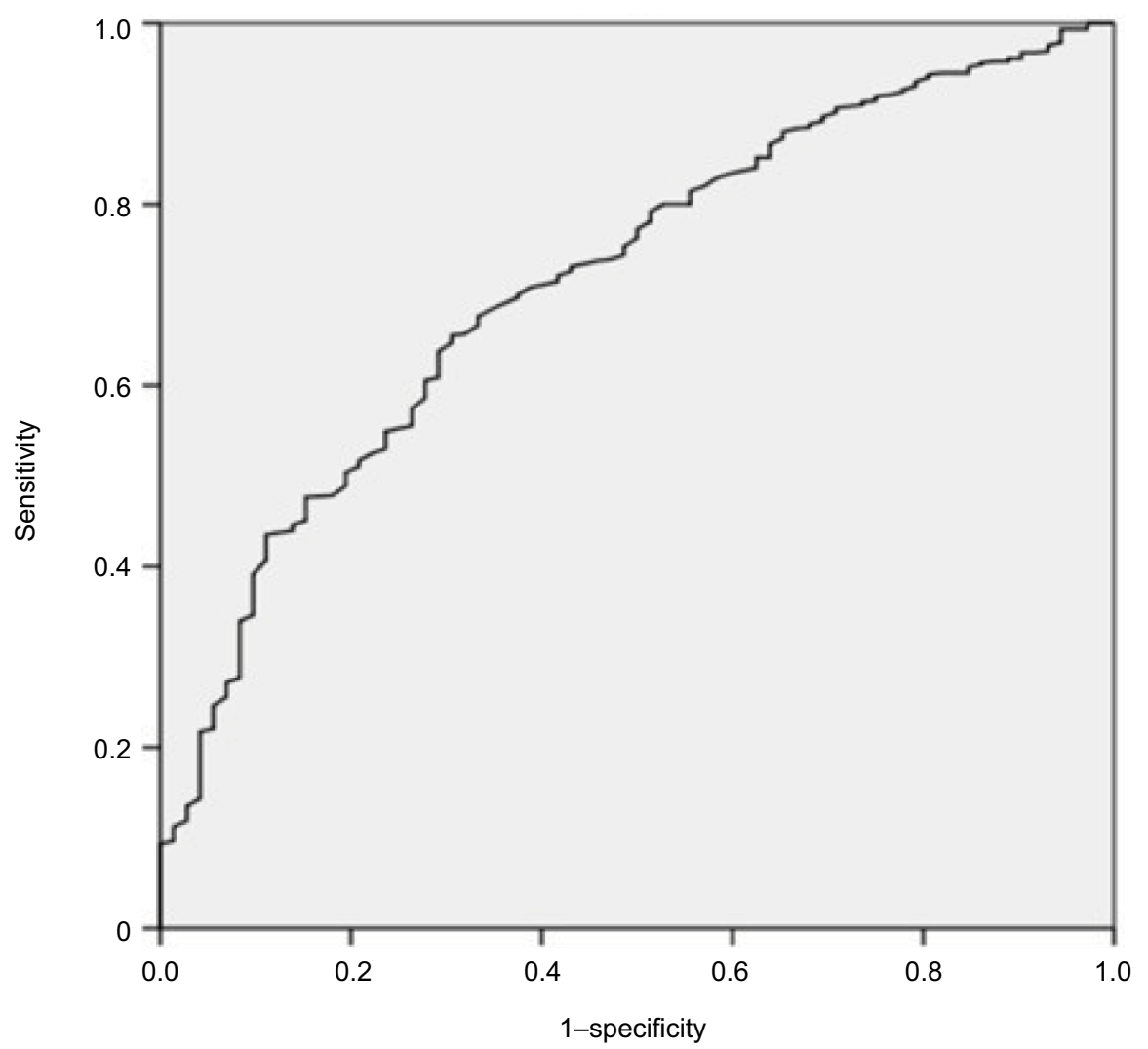

Figure I ROC analysis of skin hydration according to the presence of fissure. Note: $A \cup C=0.717 ; p<0.001$.

Abbreviations: AUC, area under the curve; ROC, receiver operating characteristic.

Table 2 Sensitivity and specificity of skin hydration for the presence of fissure

\begin{tabular}{lll}
\hline Skin hydration (\%) & Sensitivity & Specificity \\
\hline 15.0 & 0.731 & 0.569 \\
16.0 & 0.670 & 0.667 \\
17.0 & 0.628 & 0.708 \\
18.0 & 0.591 & 0.722 \\
19.0 & 0.530 & 0.764 \\
20.0 & 0.478 & 0.819 \\
21.0 & 0.425 & 0.889 \\
22.0 & 0.351 & 0.903 \\
23.0 & 0.290 & 0.917 \\
24.0 & 0.230 & 0.944 \\
25.0 & 0.172 & 0.958 \\
\hline
\end{tabular}

On the other hand, autonomic neuropathy was not associated with skin hydration $<20.0 \%$, but fissures are generally considered to develop in dry skin due to decreased perspiration associated with autonomic neuropathy. ${ }^{7}$ Additionally, sweat gland function was not evaluated in the present study, though the devices which can detect nerve damage by evaluating sweat gland function might be useful. This might represent a limitation of the present study, in which autonomic neuropathy was judged based solely on CVRR. In addition, angiopathy was not a factor associated with skin hydration $<20.0 \%$, but has been identified as a factor associated with deep fissure in patients with diabetes. ${ }^{14}$ This result might suggest that angiopathy influences deep tissues only, not superficial tissues such as the stratum corneum.

\section{Limitations of the study}

Some limitations of this study need to be considered. Lower skin hydration cannot be confirmed as a cause of fissure in this study, given the cross-sectional design. Application of moisturizer is recommended for dry skin by several guidelines, but a longitudinal study was not ethically feasible. However, lower skin hydration seems unlikely to result from fissures, because skin hydration was not measured at sites located immediately above the fissure; so, this potential flaw should have little impact on the novelty of these findings. The data about the use of moisture creams and other agents were not unavailable. Therefore, this result could not exclude the effect of this factor. 
Table 3 Factors associated with lower skin hydration (univariate)

\begin{tabular}{|c|c|c|c|}
\hline Variables & Skin hydration $<20 \%, n=383$ & Skin hydration $\geq 20 \%, n=310$ & $p$-value \\
\hline Age (years) & $66.8 \pm 10.4$ & $66.9 \pm 11.4$ & $0.929^{a}$ \\
\hline Sex & & & $<0.00 \mathrm{I}^{\mathrm{b}}$ \\
\hline Male & $242(63.1)$ & $153(49.4)$ & \\
\hline Female & $|4|(36.8)$ & $157(50.6)$ & \\
\hline Duration of diabetes (years) & $13.9 \pm 9.9$ & $14.7 \pm 9.9$ & $0.30 \mathrm{I}^{\mathrm{a}}$ \\
\hline $\mathrm{HbAlc}(\%)$ & $7.2 \pm 1.2$ & $7.2 \pm 1.1$ & $0.508^{\mathrm{a}}$ \\
\hline Autonomic neuropathyc & & & $0.788^{b}$ \\
\hline Yes & $30(7.9)$ & $26(8.4)$ & \\
\hline No & $352(92.1)$ & $283(91.6)$ & \\
\hline Angiopathy ${ }^{d}$ & & & $0.873^{\mathrm{b}}$ \\
\hline Yes & $26(6.8)$ & $20(6.5)$ & \\
\hline No & $354(93.2)$ & $286(93.5)$ & \\
\hline Monofilament test & & & $0.153^{b}$ \\
\hline Abnormal & $89(10.2)$ & $58(18.8)$ & \\
\hline Normal & $294(76.8)$ & $25 I(8 I .2)$ & \\
\hline Tinea pedis & & & $0.00 \mathrm{I}^{\mathrm{b}}$ \\
\hline Yes & $258(67.4)$ & $169(54.5)$ & \\
\hline No & $125(32.6)$ & $|4|(45.5)$ & \\
\hline Room temperature $\left({ }^{\circ} \mathrm{C}\right)$ & $24.8 \pm 2.0$ & $25.2 \pm 1.7$ & $0.005^{\mathrm{a}}$ \\
\hline Room humidity (\%) & $37.1 \pm 11.9$ & $38.7 \pm 11.9$ & $0.100^{\mathrm{a}}$ \\
\hline
\end{tabular}

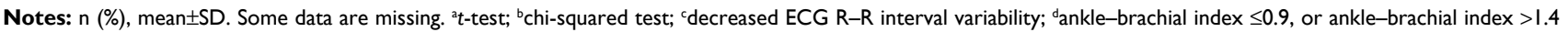
and toe-brachial index $<0.7$.

Abbreviations: ECG, electrocardiogram; HbAlc, glycated hemoglobin.

Table 4 Factors associated with lower skin hydration (multivariate)

\begin{tabular}{lllll}
\hline Variables & Odds ratio & $\mathbf{9 5 \%} \mathbf{C l}$ & p-value \\
\cline { 3 - 4 } & & Lower & Upper & \\
\hline Age (years) & 1.000 & 0.986 & 1.015 & 0.952 \\
Male sex & 1.587 & 1.157 & 2.178 & 0.004 \\
Tinea pedis & 1.548 & 1.122 & 2.135 & 0.008 \\
Room temperature & 0.900 & 0.823 & 0.985 & 0.021 \\
\hline
\end{tabular}

Notes: Logistic regression analysis. Forced entry method. Hosmer-Lemeshow analysis: $\chi^{2}=2.887 ; p=0.94$.

\section{Relevance to clinical practice}

Use of moisturizers is recommended to lubricate dry skin for patients with diabetes. ${ }^{18}$ Topical moisturizers are beneficial for managing dry skin, ${ }^{19}$ with many studies showing demonstrable improvements in skin condition compared to use of a moisturizer with placebo base cream, although no conclusive recommendations were reached in a systematic review. ${ }^{20}$ Although no particular ingredient or formulation of moisturizer could be recommended, this study revealed that application of moisturizer for prevention of fissure in patients with diabetes should be followed for a suggested skin hydration of $20.0 \%$.

\section{Conclusion}

To prevent the heel fissure, moisturizing care at a skin hydration of $<20 \%$ appears useful as a guide. Moisturizing care for preventing fissures may be particularly worthwhile for men and in cold environments. For skin hydration $<20 \%$, to prevent fissures, not only moisturizing care but also the possibility of treatment for tinea pedis should be considered.

\section{Disclosure}

The authors report no conflicts of interest in this work.

\section{References}

1. International Working Group on the Diabetic Foot, Definitions and Criteria [webpage on the Internet]. Available from: http://iwgdf.org/ guidelines/definitions-criteria-2015/. Retrieved October 1, 2017.

2. Frykberg RG, Zgonis T, Armstrong DG, et al; American College of Foot and Ankle Surgeons. Diabetic foot disorders. A clinical practice guideline (2006 revision). J Foot Ankle Surg. 2006;45(5):S1-S66.

3. Resnick HE, Carter EA, Lindsay R, et al. Relation of lower-extremity amputation to all-cause and cardiovascular disease mortality in American Indians: the Strong Heart Study. Diabetes Care. 2004;27(6):1286-1293.

4. Ragnarson Tennvall G, Apelqvist J. Health-related quality of life in patients with diabete mellitus and foot ulcers. J Diabetes Complications. 2000;14:235-241.

5. Nabuurs-Franssen MH, Huijberts MS, Nieuwenhuijzen Kruseman AC, Willems J, Schaper NC. Health-related quality of life of diabetic foot ulcer patients and their caregivers. Diabetologia. 2005;48:1906-1910.

6. International Diabetes Federation, IDF diabetes atlas [homepage on the Internet]. 7th ed. Available from: http://www.diabetesatlas.org/. Retrieved October 1, 2017.

7. International working group on the diabetic foot, Pathophysiology [webpage on the Internet]. Available from: http://iwgdf.org/consensus/ pathophysiology-of-foot-ulceration/. Retrieved October 1, 2017.

8. Pham HT, Exelbert L, Segal-Owens AC, Veves A. A prospective, randomized, controlled double-blind study of a moisturizer for xerosis of the feet in patients with diabetes. Ostomy Wound Manage. 2002;48(5):30-36. 
9. Garrigue E1, Martini J, Cousty-Pech F, Rouquier A, Degouy A. Evaluation of the moisturizer Pédimed $\left({ }^{\circledR}\right)$ in the foot care of diabetic patients. Diabetes Metab. 2011;37(4):330-335.

10. Federici A, Federici G, Milani M. Use of a urea, arginine and carnosine cream versus a standard emollient glycerol cream for treatment of severe xerosis of the feet in patients with type 2 diabetes: a randomized, 8 month, assessor-blinded, controlled trial. Curr Med Res Opin. 2015;31(6): 1063-1069.

11. Martini J, Huertas C, Turlier V, Saint-Martory C, Delarue A. Efficacy of an emollient cream in the treatment of xerosis in diabetic foot: a double-blind, randomized, vehicle-controlled clinical trial. J Eur Acad Dermatol Venereol. 2017;31(4):743-747.

12. Federici A, Federici G, Milani M. An urea, arginine and carnosine based cream (Ureadin Rx Db ISDIN) shows greater efficacy in the treatment of severe xerosis of the feet in type 2 diabetic patients in comparison with glycerol-based emollient cream. A randomized, assessor-blinded, controlled trial. BMC Dermatol. 2012; $12: 16$.

13. Seité S, Khemis A, Rougier A, Ortonne JP. Importance of treatment of skin xerosis in diabetes. J Eur Acad Dermatol Venereol. 2011;25(5):607-609.
14. Oe M, Sanada H, Nagase T, et al. Factors associated with deep foot fissures in diabetic patients: a cross-sectional observational study. Int J Nurs Stud. 2012;49(6):739-746.

15. Seino Y, Nanjo K, Tajima N, et al. Report of the Committee on the classification and diagnostic criteria of diabetes mellitus. The Committee of the Japan Diabetes Society on the diagnostic criteria of diabetes mellitus. Diabetol Int. 2010;1:2-20.

16. Burns T, Breathnach S, Cox N, Griffiths C. Rock's Textbook of Dermatology. 7th ed. Massachusetts, USA: Blackwell Science Ltd; 2004.

17. Norgren L, Hiatt WR, Dormandy JA, et al; TASC II Working Group. Inter-society consensus for the management of peripheral arterial disease. Int Angiol. 2007;26(2):81-157.

18. Schaper NC, Van Netten JJ, Apelqvist J, Lipsky BA, Bakker K; International Working Group on the Diabetic Foot (IWGDF). Prevention and management of foot problems in diabetes: a Summary Guidance for Daily Practice 2015, based on the IWGDF guidance documents. Diabetes Res Clin Pract. 2017;124:84-92.

19. Proksch E. The role of emollients in the management of diseases with chronic dry skin. Skin Pharmacol Physiol. 2008;21(2):75-80.

20. Parker J, Scharfbillig R, Jones S. Moisturisers for the treatment of foot xerosis: a systematic review. J Foot Ankle Res. 2017;7;10:9.
Chronic Wound Care Management and Research

\section{Publish your work in this journal}

Chronic Wound Care Management and Research is an international, peer reviewed, open access, online journal publishing original research, reviews, editorials, and commentaries on the causes and management of chronic wounds and the major issues related to chronic wound management. Topics also include chronic wounds as comorbidities to other

\section{Dovepress}

conditions, patient adherence to therapy, and the economic burden of chronic wounds. The manuscript management system is completely online and includes a very quick and fair peer review system, which is all easy to use. Visit http://www.dovepress.com/testimonials.php to read real quotes from published authors. 